\title{
Philosophiques
}

\section{Anne-Marie Décaillot, Cantor et la France. Correspondance du mathématicien allemand avec les Français à la fỉn du xix siècle, Paris, Éditions Kimé, 2008, 347 p.}

\section{Mark van Atten}

Volume 37, numéro 1, printemps 2010

Albert Lautman, philosophe des mathématiques

URI : https://id.erudit.org/iderudit/039730ar

DOI : https://doi.org/10.7202/039730ar

Aller au sommaire du numéro

Éditeur(s)

Société de philosophie du Québec

ISSN

0316-2923 (imprimé)

1492-1391 (numérique)

Découvrir la revue

Citer ce compte rendu

van Atten, M. (2010). Compte rendu de [Anne-Marie Décaillot, Cantor et la

France. Correspondance du mathématicien allemand avec les Français à la fin du xix ${ }^{e}$ siècle, Paris, Éditions Kimé, 2008, 347 p.] Philosophiques, 37(1), 262-265.

https://doi.org/10.7202/039730ar d'utilisation que vous pouvez consulter en ligne.

https://apropos.erudit.org/fr/usagers/politique-dutilisation/ 
l'analyse des théories mathématiques, alors que l'étude de la pratique mathématique est essentielle pour Peirce.

Le mérite principal de cet ouvrage est, à mon avis, de proposer sans doute la meilleure analyse comparative jamais réalisée de la philosophie des mathématiques de Peirce par rapport à celles de Kant, Frege, Wittgenstein et Hintikka, tout en apportant des éclaircissements très intéressants pour situer Peirce par rapport au platonisme mathématique.

JÉRÔME HAVENEL

Université du Québec à Montréal

\begin{abstract}
Anne-Marie Décaillot, Cantor et la France. Correspondance du mathématicien allemand avec les Français à la fin du XIX siècle, Paris, Éditions Kimé, 2008, 347 p.
\end{abstract}

L'ouvrage de Décaillot est construit autour des chapitres suivants: 1. Les sources; 2. Les combats de Cantor; 3. Des intellectuels catholiques aux occultistes. Une étonnante diversité de rélations; 4. À la recherche d'une harmonie entre science et foi (théologie, philosophie et mathématique); 5. Cantor et la conjecture de Goldbach; 6 . Est beau ce qui est beau intérieurement ; 7. La correspondance. L'ouvrage comporte également quatre appendices: 1. Liste des lettres de Cantor aux Français; 2. Les différents sens de l'expression «infini mathématique»; 3 . La genèse de la théorie cantorienne des ensembles; 4 . Calculs probabilistes en théorie des nombres. Il peut être conseillé de procéder d'abord à la lecture des lettres (ce qui peut aisément se faire d'un seul coup si on ne les lit que dans une seule langue), pour ensuite se tourner vers les chapitres thématiques détaillés de l'auteur pour de l'information d'arrière-plan.

Les trente-sept lettres et esquisses composant cet ensemble sont présentées dans un ordre chronologique et s'étendent sur une période de dix ans, entre 1886 et 1896, commençant ainsi à une période où Cantor avait réalisé la plus grande partie de son travail en théorie des ensembles, tout juste après sa première dépression en 1884 , et jusqu'un peu avant sa deuxième dépression de 1899. Toutes les lettres sont de Cantor, à l'exception d'une qui lui est adressée.

Ses quatorze correspondants sont les mathématiciens Charles Hermite, Camille Jordan, Henri Poincaré, Jules Tannery, les intellectuels catholiques Élie Blanc, Maurice d'Hulst, Claude Alphonse Valson; les polytechniciens Charles-Ange Laisant et Émile Lemoine; le philosophe et historien des sciences Paul Tannery: l'occultiste Papus (Gérard Encausse); le scientifique Charles Henry, le sénateur Barthélémy Saint-Hilaire, ainsi que le fondateur de la Revue de métaphysique et de morale, Xavier Léon. 
Dans la plupart des cas, ce ne sont pas les lettres qui ont été effectivement reçues par les correspondants de Cantor qui sont présentées dans l'ouvrage, mais plutôt les esquisses de ces lettres qu'a conservé Cantor, préservées aujourd'hui aux Archives Cantor de la Niedersächsische Staats-und Universitätsbibliothek de Göttingen. Toutefois, lorsque la lettre originale était disponible dans les archives ou les publications du correspondant, c'est celle-ci qui a été publiée (comme dans le cas des lettres à Jordan, Paul Tannery et Poincaré), et les différences entre l'original et l'esquisse sont répertoriées en notes. De chaque lettre, l'original allemand est présenté, suivi de la traduction française de Décaillot et ses notes. Seul un petit nombre de ces lettres ont été publiées, en tout ou en partie, dans les éditions allemandes Georg Cantor: Briefe (dirigé par Herbert Meschowski et Winfried Nilson en 1991) et dans Kardinalität und Kardinale (dirigé par Christian Tapp en 2005). Il aurait été souhaitable que l'auteure fasse mention des lettres qui ont été publiées ailleurs. Comme elle l'indique, la correspondance de Cantor avec les Français doit avoir été plus importante que ce que suggère le nombre d'esquisses qui nous sont restées.

La variété des correspondants ainsi que des sujets mentionnés dans les titres de chapitre reflète le large registre des intérêts de Cantor. Toutefois, les lettres rassemblées dans cet ouvrage n'ont pas été, pour la plupart, un véhicule que Cantor aurait utilisé pour développer ses idées mathématiques et philosophiques (à propos desquelles la correspondance contient toutefois quelques affirmations lapidaires). En ce sens, les lettres rassemblées sont de nature différente de celles qui forment sa correspondance avec Dedekind par exemple, ou celle avec plusieurs cardinaux. Étant donné cette diversité, un titre plus approprié à ce volume, du moins à première vue, aurait été "Cantor et ses correspondants français", et l'auteure m'a informé avoir considéré cette possibilité. Pourtant, son choix définitif d'un titre suggérant un fort aspect national est entièrement justifié. Car après tout, il y a un thème qui encadre toute cette correspondance, et c'est bien celui des efforts de Cantor à obtenir en France des supports à son projet de réunir les communautés française et allemande de mathématiciens après la guerre de 1870. Le fait que la conférence de Cantor au congrès de Caen de 1894 (organisé par l'Association française pour l'avancement des sciences, dont la devise était "Pour la science, pour la patrie »), soit la seule conférence d'un mathématicien allemand en France entre 1872 et 1914, est un bon indicateur de la rupture entre les deux pays.

Grâce à l'idéalisme et au talent diplomatique de Cantor, égalant celui de ses partenaires français et à celui de Mittag-Leffler en Suède, cet effort de réconciliation a été un succès complet et résulta d'abord en un congrès préparatoire à Zürich en 1897, et ensuite au premier congrès international des mathématiciens à Paris en 1900. Cantor a agi en coulisses; par exemple, sa signature n'apparaît pas parmi celles qu'on retrouve sur les invitations au congrès. Une des raisons qui explique sa volonté de rester discret était sa 
relation problématique avec l'establishment académique allemand. En effet, Cantor ne se sentait à l'aise ni avec la hiérarchie académique allemande, dans laquelle sa position était relativement faible, ni non plus dans le style de mathématiques qui était préconisé. Se voyant comme le défenseur des «mathématiques libres» contre les «mandarins de Berlin» (l'école de Weierstrass, de laquelle il avait reçu la plupart de sa formation en mathématiques), il dirigea ses énergies ailleurs, et de manière notable vers l'Association des mathématiciens allemands (Deutsche Mathematiker-Vereinigung), dont il avait contribué à la fondation et qui était en faveur de rétablir des liens internationaux), ainsi que vers ses échanges épistolaires avec ses correspondants en Allemagne et à l'étranger. Dans sa correspondance avec les Français, il a su retourner son isolation de l'establishment académique allemand et les préjudices à son endroit à son avantage, en mettant de plus l'accent sur le fait qu'il était né à Saint-Pétersbourg et que ses racines étaient dano-russes plutôt qu'allemandes (voir en particulier la lettre 22 à Hermite, la lettre 27 à Poincaré et la lettre 28 à Paul Tannery: une copie numérique du manuscrit original de la lettre 27 est accessible en ligne dans un article de Décaillot sur http://images.math.cnrs.fr/L-entente-cordiale-scientifique.html). Le parallèle entre l'isolation de Cantor et celui de la France se fait constamment sentir dans cette correspondance.

Un thème mathématique intéressant dans les lettres est la tentative de Cantor d'établir la preuve de la conjecture de Goldbach. L'auteure soulève la question de savoir si cette tâche aurait pu en fin de compte être motivée par un souhait de trouver des applications à sa théorie des ensembles alors nouvelle vers une théorie des nombres. Des douze lettres connues dans lesquelles Cantor discute cette conjecture (1885-1896), sept sont adressées à ses correspondants français Hermite, Laisant et Lemoine (p. 124). Cantor avait vérifié la conjecture pour les nombres pairs jusqu'à 1000, et avait de plus calculé le nombre de décompositions en deux nombres primes qu'ils ont. (Cette vérification était le sujet de sa contribution au congrès de Caen que nous avons déjà mentionné.) Ses observations sur le nombre de décompositions ont mené Cantor à formuler deux conjectures; toutes les deux se sont révélées fausses. L'auteure discute de ces conjectures à la lumière des travaux plus tardifs de Hardy et Littlewood.

Un mathématicien français dont l'absence est remarquable dans ce volume, et avec qui d'ailleurs Cantor ne semble jamais avoir eu de contact, est Charles Méray (1835-1911: Cantor est né en 1845 et mort en 1918). Méray avait publié l'idée d'éliminer les limites problématiques des séries infinies convergentes de rationnels, préférant travailler seulement avec les séries elles-mêmes, et ce, même avant que Cantor et Heine le fissent (cependant, contrairement à eux, il s'est gardé de les appeler des "nombres", conservant le concept euclidien de nombre comme agrégat d'unités.).

À mon avis, ce qui fait défaut dans la contextualisation de la correspondance offerte par l'auteure est le boycott international, immédiatement 
après la Première Guerre mondiale, des scientifiques issus des pays des Empires centraux. Alors que Cantor lui-même mourut en janvier 1918, ce boycott et les difficultés à le surmonter méritent aussi bien d'être discutés que ne le méritent les développement plus tardifs en théorie des ensembles ou autour de la conjecture de Goldbach, deux thèmes commentés par l'auteure. Il faut également souligner que Mittag-Leffler (1846-1927), l'ami de Cantor qui, de 1873 à 1876, fit des séjours en France (auprès de Poincaré et Herminte) et en Allemagne (auprès de Weierstrass), fut très actif non seulement après la guerre franco-prussienne, mais aussi après la Première Guerre mondiale dans ses tentatives de restaurer les contacts et l'harmonie entre les mathématiciens des États autrefois en conflit.

Avec la traduction française, en 1962, de la correspondance CantorDedekind telle qu'éditée par Cavaillès et Noether en 1937, cet ouvrage est la première traduction substantielle des lettres de Cantor en français. Les présentes remarques ne pourront rendre justice aux riches et utiles introductions de l'auteure. Il est à espérer qu'elle poursuive ce précieux travail.

MARK VAN ATTEN

Institut d'Histoire et de Philosophie des Sciences et des Techniques (CNRS/Paris 1/ENS) Paris

Isabelle Thomas-Fogiel, Le concept et le lieu. Figures de la relation entre art et philosophie, Paris, Cerf, «La nuit surveillée», 2008, $377 \mathrm{p}$.

L'auteure de ces pages a fait paraître aux éditions du Cerf des traductions de Cassirer et de Fichte (2005-2006) et chez Vrin, Présentation de la doctrine de la science (1999), Critique de la représentation (2000), Fichte, réflexion et argumentation (2004), Référence et auroréférence (2006). Professeure agrégée de philosophie à l'Université d'Ottawa, Isabelle Thomas-Fogiel occupait tout récemment (2007-2009) la chaire d'études de la France contemporaine au Cérium de l'Université de Montréal. Au premier coup d'œil, son ouvrage semble s'ancrer dans la problématique du statut philosophique de l'esthétique (M. Devereaux). Au milieu des années 90 et sur un autre continent, R. Rochlitz avait distingué, dans ses discussions avec J.-M. Schaeffer, entre esthétique philosophique, histoire de l'art et critique d'art. Mais l'esthétique ne se confond pas avec la philosophie de l'art et n'épuise pas non plus le champ théorique, ajoutait de son côté A. Cauquelin, tandis que $\mathrm{D}$. Chateau envisageait de plein front les questions territoriales. Dans sa rétrospective des idées esthétiques, $\mathrm{M}$. Seel avait évoqué auparavant deux tendances: la «surenchère» et la "conception privative» des œuvres d'art. L'auteure prétend, pour sa part, ne faire ni philosophie de l'art ni verser dans les définitions, et avoue plutôt s'interroger sur les spécificités et dialectiques en se concentrant sur la relation entre art et philosophie, laquelle présente 